\title{
Effects of Rome IV Definitions of Functional Dyspepsia Subgroups in Secondary Care
}

K. Van den Houte, ${ }^{*}$ Florencia Carbone, ${ }^{*}$ Nick Goelen, ${ }^{*}$ Jolien Schol, ${ }^{*}$ Imke Masuy, Joris Arts, ${ }^{\ddagger}$ Philip Caenepeel, ${ }^{\S}$ Dirk Staessen, " Philippe Vergauwe," Guy Van Roey," Pascale Latour,-" Hubert Piessevaux,"- Philippe Maldague," Ariane Gerkens,"- Fabien Wuestenberghs, "1ๆ Alain Vandenberghe,"\# and Jan Tack*

${ }^{*}$ Translational Research Center for Gastrointestinal Diseases, University of Leuven, Leuven, Belgium; ${ }^{\ddagger}$ Maagdarm en Leverziekten, Algemeen Ziekenhuis Sint Lucas, Brugge, Belgium; §'Ziekenhuis Oost-Limburg, Genk, Belgium; "Sint Vincentius Ziekenhuis, Antwerpen, Belgium; " Dienst Gastro Enterologie, AZ Groeninge, Kortrijk, Belgium; "Department of Gastroenterology, Sint Elisabeth Ziekenhuis, Turnhout, Belgium; ${ }^{* *}$ Department of Gastroenterology, University Hospital CHU of Liege, Liege, Belgium; ${ }^{\ddagger \neq}$ Department of Gastroenterology and Hepatology, Cliniques Universitaires St-Luc, Université

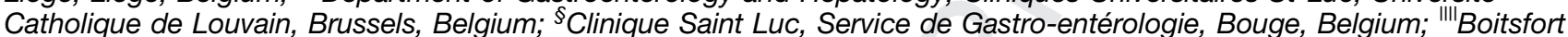
Medical Center, Brussels, Belgium; " Department of Gastroenterology and Hepatology, CHU UCL Namur, Godinne University Hospital, UC Louvain, Yvoir, Belgium; "\# Medical Research Consultant, BVBA, Chaumont-Gistoux, Belgium

BACKGROUND \& AIMS:

Functional dyspepsia (FD) is subdivided into postprandial distress syndrome (PDS) and epigastric pain syndrome (EPS) according to the Rome III consensus. In clinical practice, there is a major overlap between these subgroups. The Rome IV criteria included postprandially occurring symptoms in the PDS subgroup. We aimed to analyze the effects of the Rome IV criteria, compared with Rome III, on FD subgroups in patients recruited from secondary care.

METHODS:

Patients with FD ( $n=224$; mean age, $43 \pm 1$ y; 77\% women) were recruited from secondarycare units in Belgium and filled out symptom questionnaires, allowing subdivision according to Rome III and Rome IV criteria and identification of postprandial symptoms. Symptom patterns and demographics were compared between the subgroups. Statistical analysis was performed using the $t$ test and the Fisher exact test.

RESULTS: $\quad$ According to the Rome III criteria, $25 \%$ of participants had PDS, $8 \%$ had EPS, and $67 \%$ had an overlap. Postprandial fullness, early satiation, and bloating were present in significantly more patients in the PDS and overlap groups than the EPS group $(P<.0001)$. A higher proportion of patients in the overlap group showed symptoms such as postprandial epigastric pain and nausea than in the EPS group (both $P \leq .02$ ). With the Rome IV criteria, the overlap group was reduced to $35 \%$; $57 \%$ of patients were considered to have PDS and $8 \%$ to have EPS. Postprandial pain was significantly more prevalent in the PDS than in the EPS group $(P \leq .002)$, and postprandial nausea was significantly more prevalent in the PDS group than the overlap group $(P=.007)$. overlap between PDS and EPS groups. Studies are needed to determine if Rome IV subgroups are associated differently with psychological comorbidities and treatment responses. 
unctional dyspepsia (FD) is one of the most com$\mathbf{H}$ mon functional gastrointestinal disorders, with estimates of up to $21 \%$ population prevalence. ${ }^{1,2}$ The Rome III consensus defined FD as a condition characterized by symptoms that were thought to originate from the gastroduodenal segment in the absence of an organic, systemic, or metabolic disease likely to explain the symptoms. ${ }^{3}$ In this consensus, only 4 symptoms were considered as cardinal FD symptoms: postprandial fullness, early satiation, epigastric pain, and epigastric burning. In addition, to optimize the management of FD, the Rome III consensus proposed a subdivision into meal-related FD or postprandial distress syndrome (PDS) and meal-unrelated FD or epigastric pain syndrome (EPS). ${ }^{3,4}$ PDS comprises FD patients who experience bothersome postprandial fullness after ordinarysized meals occurring at least several times a week and/or early satiation that prevents finishing a regular meal at least several times a week. EPS includes patients experiencing epigastric pain or burning at least once a week. The pain is intermittent, not generalized or localized to other abdominal or chest regions, not relieved by defecation or passage of flatus, and not related to gallbladder or sphincter of Oddi disorders.

Epidemiologic studies after the Rome III consensus confirmed that both EPS and PDS existed as separate entities in the general population, with a minority of subjects in the overlap PDS-EPS group. ${ }^{4-7}$ However, in clinical practice, the separation between EPS and PDS was less clear, and the majority of patients was found in the overlap group presenting with both EPS and PDS symptoms, which of course hampers the applicability of the subdivision for clinical management. ${ }^{4,8-10}$ Although the PDS subgroup was defined as displaying "meal-related dyspeptic symptoms," it focused only on postprandial fullness and early satiation. Several clinical observations showed that many FD patients report postprandially occurring epigastric pain or nausea. ${ }^{11-14}$ When postprandial non-PDS symptoms such as epigastric pain and postprandial nausea are considered part of an "adapted" PDS group, a better separation from EPS is obtained. ${ }^{14}$ These principles have been applied to the Rome IV definitions, in which PDS now refers to all meal-related symptoms, to consider not only bothersome postprandial fullness and bothersome early satiation, but also postprandial epigastric pain or burning, epigastric bloating, excessive belching, and nausea as part of the same spectrum. ${ }^{15}$ EPS still is defined as bothersome epigastric pain and/or bothersome epigastric burning, occurring at least 1 day a week. ${ }^{15}$ Based on our analysis, conducted on a secondary care patient population before the Rome IV consensus, this approach indeed has the potential to decrease the overlap between EPS and PDS. An analysis of prevalence of functional gastrointestinal disorders in the general population in 3 countries also supports this notion, ${ }^{16}$ but data from clinical practice are lacking.

\section{What You Need to Know}

\section{Background}

The Rome III consensus subdivided functional dyspepsia into postprandial distress syndrome (PDS) and epigastric pain syndrome (EPS). In clinical practice, using the Rome III subdivision results in overlap between PDS and EPS.

\section{Findings}

When the Rome IV subdivision is used, the overlap between PDS and EPS is limited. The decrease of the overlap group is paralleled by an increase in the PDS group. The PDS group is the largest subgroup in the Rome IV classification.

\section{Implications for patient care}

The Rome IV criteria should be used for analysis of patients with functional dyspepsia.

The aim of this study was to evaluate the impact of the Rome IV criteria on the PDS and EPS subgroups and their overlap in secondary care, and in comparison with Rome III criteria. We hypothesize a better division in different FD subgroups with a lower percentage of patients in the overlap group and a higher proportion in the PDS subgroup based on Rome IV criteria, compared with Rome III.

\section{Materials and Methods}

\section{Patients}

Patients, ages between 18 and 70 years old, presenting with dyspeptic symptoms were recruited from 8 gastroenterology secondary care sites in Belgium. Patients referred by their general practitioner to secondary care gastroenterology specialists or care levels, for epigastric symptoms with a negative endoscopy, filled out a symptom questionnaire. Helicobacter pylori-positive patients or those receiving treatment for $H$ pylori eradication during the past 3 months were excluded from the study. Furthermore, patients with diabetes mellitus, with a confirmed organic gastrointestinal disorder or a concomitant major organic condition that may explain their digestive symptoms, or females who were pregnant or lactating were ineligible to participate. Patients presenting with predominant symptoms of irritable bowel syndrome, daily symptoms of nausea, vomiting more than 1 day a month, daily symptoms of excessive belching, and predominant symptoms of gastroesophageal reflux disease were excluded. Furthermore, patients with a former digestive surgery affecting upper gut motility could not participate. The Research Ethics Committee UZ/KU Leuven Q15 approved this study and informed consent was obtained from all patients before any study procedures 
were performed. The study was performed in accordance with the 1975 Declaration of Helsinki and the BMJ guidelines.

\section{Study Design}

Patients with epigastric symptoms and a negative endoscopy at secondary care sites in Belgium filled out an adapted Rome III gastroduodenal questionnaire that contained additional questions regarding the relationship of symptoms and meals, allowing Rome IV subgroup diagnoses. Questions were available in Dutch or French according to the mother tongue of the patient.

\section{Data Analysis}

After filling out the questionnaire, patients were subdivided into pure PDS, pure EPS, and the overlapping EDS-PDS subgroups as per Rome III criteria for FD. The group with PDS was defined by postprandial fullness and/or early satiety. The EPS subgroup was characterized by epigastric pain and burning occurring at least several times a week. The occurrence and frequency of symptoms were compared between all subgroups.

A second analysis was performed on the same set of patients based on the Rome IV consensus to include patients with postprandial symptoms of nausea and postprandial epigastric pain within the PDS subgroup. The presence and frequency of symptoms were analyzed and compared with the subgroups defined by Rome III.

\section{Statistical Analysis}

The presence and severity of symptoms were defined and proportions of patients with symptoms were compared using the Fisher exact test. Results are shown as means $\pm \mathrm{SD}$. Nonsignificant results are indicated as NS. Statistical analyses were performed using GraphPad. $P$ values less than .05 were considered significant.

\section{Results}

\section{Patient Selection}

In this trial, 250 secondary care level dyspeptic patients were recruited from 8 sites in Belgium. The mean age of the patients was $43 \pm 1$ years and $77 \%$ were women. Twenty-six patients did not meet the Rome criteria for FD, and were characterized by symptoms such as bloating $(23 \%)$, nausea $(5 \%)$, and belching (15\%). These patients were excluded from the analysis, resulting in 224 FD patients used for analysis.

\section{Symptom Patterns}

Ninety-one percent of all patients reported postprandial fullness at least several times a week. In addition, $76 \%$ of all patients reported bloating at least several times a week, followed by epigastric pain at least once a week (72\%). Early satiation and epigastric burning were present in 58\% and 38\%, respectively, of all patients.

\section{Subdivision According to Rome III Criteria}

Using the Rome III criteria, $25 \%$ of the patients were classified as pure PDS (postprandial fullness and/or early satiation several times a week with epigastric pain or burning occurring less than once a week) (Figure 1). Their mean age was $43 \pm 3$ years and $70 \%$ were women. Eight percent were considered pure EPS (epigastric burning and/or pain at least once a week without relevant postprandial fullness or early satiation) with a mean age of $49 \pm 4$ years and $64 \%$ female predominance. Overlapping PDS-EPS, the largest subgroup, was found in $67 \%$ of all patients ( $42 \pm 1 \mathrm{y} ; 81 \%$ women).

As expected, the main symptoms of the Rome III FD-PDS group were postprandial fullness and early satiation at least several times a week, present in $96 \%$ and $69 \%$, respectively. In addition, $77 \%$ of the PDS subgroup experienced bloating at least several times a week. In line with the Rome III subgroup definitions, postprandial fullness, early satiation, and bloating were reported significantly more frequently in the PDS and overlap groups (99\% postprandial fullness, $60 \%$ early satiation, and $80 \%$ bloating) compared with the EPS group (0\%, 0\%, and 27\%, respectively; all $P<.001)$. The Rome III EPS group was characterized by epigastric pain $(72 \%)$, which occurred significantly less frequently than in the Rome III overlap group (95\%; $P=.006)$. Epigastric burning was present in $56 \%$ of the EPS group and in $48 \%$ of the overlap group (NS). In addition, postprandial ${ }^{17}$ pain was reported more often by the Rome III overlap group (76\%) compared with the PDS and EPS groups (25\% and $12 \%$, respectively; $P<.001$ ).

Other reported gastrointestinal symptoms were nausea and belching. Nausea occurred at least several times a week in $36 \%$ of the PDS group and in $40 \%$ of the overlap group, which was significantly higher than in the EPS group $(11 \% ; P=.02)$. Belching appeared in a similar amount of patients in all groups (28\% EPS, 25\% PDS, $32 \%$ overlap; NS).

\section{Subdivision According to Rome IV Criteria}

In a second analysis using the Rome IV definition, postprandially occurring epigastric pain was considered a symptom of the PDS group. All patients were divided into the 3 subgroups: PDS (57\%; mean age, $42 \pm 1.5 \mathrm{y}$; $78 \%$ women), EPS (8\%; mean age, $49 \pm 4.1$ y; $64 \%$ women), and the overlap EPS-PDS group (35\%; mean age, $42 \pm 2.0$ y; $78 \%$ women).

Within the PDS group, 98\% reported postprandial fullness, which was similar to $99 \%$ in the overlap group 

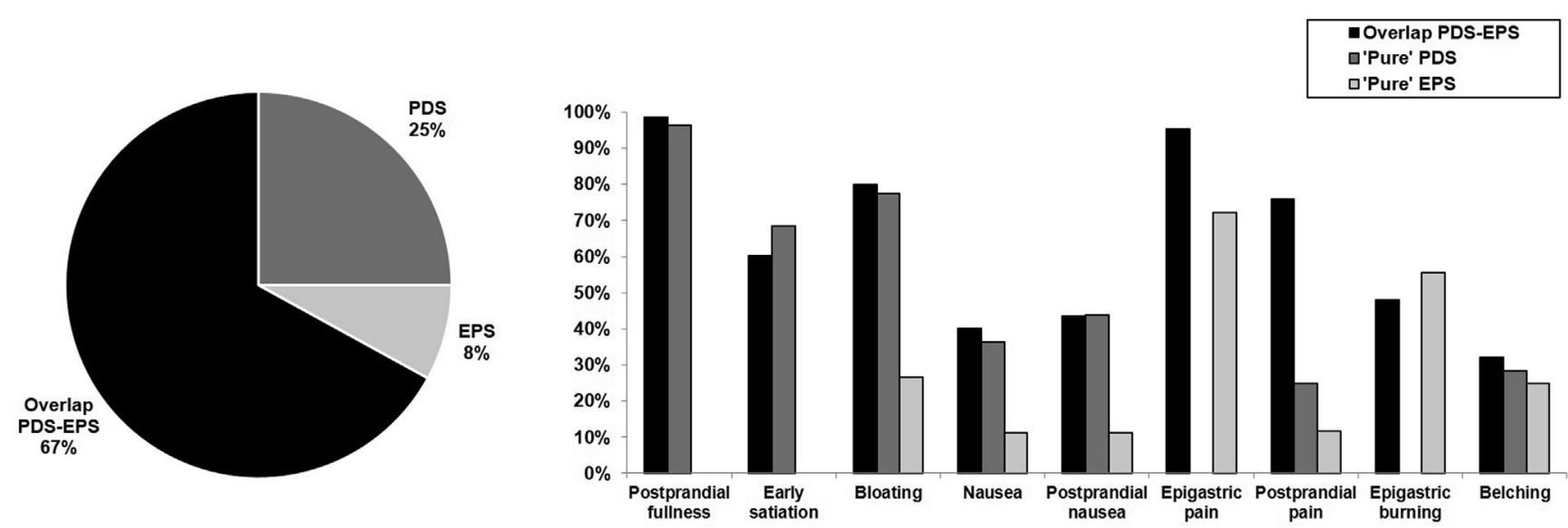

Figure 1. Subgroup prevalence (upper panel) and symptom characteristics (lower panel) when applying the Rome III definitions Q18 for epigastric pain syndrome (EPS) and postprandial distress syndrome (PDS).

(NS). Meanwhile, early satiation was reported significantly less frequently in the overlap group compared with the PDS group (49\% vs $71 \%$; $P=.003)$. PDS patients were characterized by a lower occurrence of epigastric pain compared with the overlap group (59 vs 91\%; $P<.001$ ). No significant difference was found in comparison with the EPS group (72\%). Postprandial epigastric pain was reported less frequently by the EPS group $(12 \%)$ compared with the PDS group $(72 \%$; $P<$ $.001)$ and the overlap group (53\%; $P=.002)$. Epigastric burning was present in $27 \%, 56 \%$, and $52 \%$ of the PDS, EPS, and overlap groups, respectively, with significant differences between PDS and EPS $(P=.01)$ and the overlap $(P<.001)$ groups.

In addition, the prevalence of upper abdominal bloating was higher in PDS and overlap patients $(85 \%$ and $71 \%$ ) compared with the EPS group $(27 \% ; P<.001$ for both). The symptom occurrence rating for belching was similar in all groups, but the EPS group reported less nausea than the PDS and overlap patients $(11 \%$ vs $40 \%$, $P=.02$; vs $37 \%, P=.05$; respectively). However, postprandial nausea was reported more often in PDS patients compared with the overlap and EPS groups (51\% vs $31 \%, P=.007$; vs $11 \%, P=.002$, respectively).

The profiles of symptoms of all groups according to the Rome IV criteria are presented in Figure 2.

\section{Discussion}

The management of patients with FD, one of the most common gastrointestinal disorders, is hampered by the lack of treatments of proven efficacy. ${ }^{15,17-19}$ It often has been argued that FD is a heterogeneous condition, with variable underlying pathophysiology, and that this explains why it is so difficult to develop generally effective treatment approaches. ${ }^{20,21}$ Already since the earliest Rome classifications FD subdivisions have been proposed, but most of them did not persist as epidemiologic, therapeutic, and scientific knowledge evolved., ${ }^{422-24}$
The Rome III consensus and its subdivisions into EPS and PDS generated a radical change from previous definitions, narrowing down FD to 4 cardinal symptoms. ${ }^{3}$ Epidemiologic studies confirmed the existence of EPS and PDS as defined by Rome III as separate entities in the general population, with modest overlap. ${ }^{4-7}$ However, in clinic samples, the overlap group was dominantly the most prevalent one. ${ }^{4,8-10,14}$ The Rome IV consensus adapted the PDS and EPS definitions, aiming to diminish the overlap by consistently considering meal-related symptoms as PDS, regardless of the nature of the symptom. $^{15}$

We analyzed the impact of this change on the classification and symptom pattern of 224 FD patients recruited from 8 secondary level care settings in Belgium. As expected, based on the known FD epidemiology, ${ }^{1-7,15,16}$ patients were on average in their early 40 s and were predominantly women.

Using the Rome III definition, we confirmed earlier reports that the largest subgroup was the PDS-EPS overlap group (67\%), followed by PDS alone $(25 \%)$ and EPS alone $(8 \%)$. If the management of patients should be based on the FD subgroup, then the overlap group poses a major challenge and uncertainty: should they be treated initially as PDS, or as EPS, or should treatment for each of these entities be combined at the offset?

We already published that the overlap within the Rome III subdivision is reduced significantly when postprandial symptoms are considered to represent PDS. ${ }^{14}$ This was implemented in the Rome IV criteria, and in a previous study by our group in tertiary care FD patients referred for gastric emptying testing we confirmed that, using Rome IV, the overlap group is reduced significantly, the pure PDS group becomes the dominant group, and the size of the pure EPS group is unchanged. ${ }^{25}$ Similar findings also were reported at the general population level in a study in the United States, Canada, and the United Kingdom. ${ }^{16}$ With the present study, which showed the same in a secondary care FD 

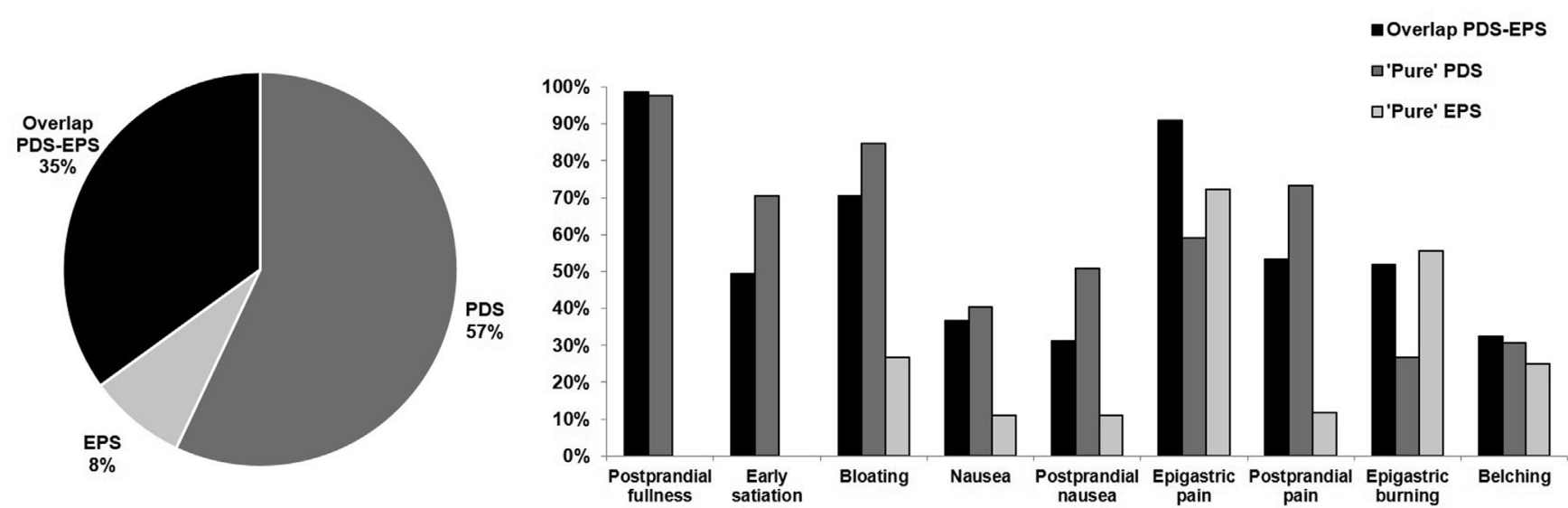

Figure 2. Subgroup prevalence (upper pane/) and symptom characteristics (lower pane/) when applying the Rome IV definitions Q19 for epigastric pain syndrome (EPS) and postprandial distress syndrome (PDS).

population, it seems valid to state that the reduced overlap group and the enlarged PDS group are common effects of the Rome IV adaptation from Rome III.

Reducing the overlap group is not a goal in itself. One goal is to identify subgroups with a more homogeneous underlying pathophysiology. Our own study found no difference in the prevalence of impaired gastric accommodation, delayed gastric emptying, or hypersensitivity to gastric distention when FD patients were subdivided according to the Rome III consensus. ${ }^{12}$ Other investigators reported similar findings. ${ }^{26,27}$ Whether a better separation of pathophysiological mechanisms can be obtained with the Rome IV approach remains to be studied.

A second goal is to identify subgroups with different treatment responses. The Rome III consensus proposed that the EPS group might respond best to proton pump inhibitors and the PDS group might respond best to prokinetic agents. ${ }^{3,17-19}$ However, this was confirmed only partially in prospective studies. ${ }^{28,29}$ Few studies have evaluated treatment responses in FD subgroups according to the Rome IV definitions. In a preliminary report of a placebo-controlled study with itopride, the best response was observed in the overlap group rather than in the pure PDS group according to Rome III. ${ }^{30}$ The features of this group seem to correspond to what now would be PDS according to Rome IV, with postprandial pain being part of the Rome IV PDS spectrum. ${ }^{15}$ Of course, many more studies are needed to substantiate the claim of superior clinical relevance of the Rome IV subgrouping.

Besides the clear need to study the treatment response of the Rome IV PDS group further, the not inconsiderable overlap group according to Rome IV, comprising approximately a third of the patients in the current study, also needs to be analyzed in depth in terms of comorbidity pattern and treatment response. This group has high prevalences of upper abdominal bloating and nausea, similar to those found in the pure PDS group and higher than in the Rome IV EPS group. These associations suggest that the overlap group according to Rome IV may in fact be more reminiscent of

Table 1. Demographic and Symptom Characteristics of the Patient Population Subdivided According to Rome III or Rome IV Q20 Definitions

\begin{tabular}{|c|c|c|c|c|c|c|}
\hline \multirow[b]{2}{*}{ Subgroup } & \multicolumn{3}{|c|}{ Rome III } & \multicolumn{3}{|c|}{ Rome IV } \\
\hline & Overlap & PDS & EPS & Overlap & PDS & EPS \\
\hline Proportion & $67 \%$ & $25 \%$ & $8 \%$ & $35 \%$ & $57 \%$ & $8 \%$ \\
\hline Female sex & $81 \%$ & $70 \%$ & $64 \%$ & $78 \%$ & $78 \%$ & $64 \%$ \\
\hline Age, $y$ & $42 \pm 1$ & $43 \pm 3$ & $49 \pm 4$ & $42 \pm 2$ & $42 \pm 2$ & $49 \pm 4$ \\
\hline \multicolumn{7}{|l|}{ Symptom frequency } \\
\hline Postprandial fullness & $99 \%$ & $96 \%$ & $0 \%$ & $99 \%$ & $98 \%$ & $0 \%$ \\
\hline Early satiation & $60 \%$ & $69 \%$ & $0 \%$ & $49 \%$ & $71 \%$ & $0 \%$ \\
\hline Bloating & $80 \%$ & $77 \%$ & $27 \%$ & $71 \%$ & $85 \%$ & $27 \%$ \\
\hline Nausea & $40 \%$ & $36 \%$ & $11 \%$ & $37 \%$ & $40 \%$ & $11 \%$ \\
\hline Epigastric pain & $44 \%$ & $0 \%$ & $72 \%$ & $91 \%$ & $59 \%$ & $72 \%$ \\
\hline Postprandial pain & $76 \%$ & $25 \%$ & $12 \%$ & $53 \%$ & $73 \%$ & $12 \%$ \\
\hline Epigastric burning & $48 \%$ & $0 \%$ & $56 \%$ & $52 \%$ & $27 \%$ & $56 \%$ \\
\hline Belching & $32 \%$ & $28 \%$ & $25 \%$ & $32 \%$ & $31 \%$ & $25 \%$ \\
\hline
\end{tabular}

EPS, epigastric pain syndrome; PDS, postprandial distress syndrome. 
PDS than EPS. Although it is not inconceivable that these patients also will respond to prokinetics, it also is possible that additional features, such as the predominant symptom, ${ }^{24}$ weight loss, ${ }^{2,21}$ or of psychosocial comorbidities $^{3,5,15}$ will determine treatment responses. Prospective treatment outcome studies should evaluate these aspects in FD subgroups according to the Rome IV consensus.

Strengths of this study were the setting at the secondary care level, based on referral from general practitioners, and the use of a single questionnaire across different practices. Limitations were the relatively limited sample size in comparison with epidemiologic studies, ${ }^{2,10}$ and the setting where data were collected was only in Belgium. A final limitation was the exclusion of $H$ pylori-infected patients. However, recent studies have shown that these are only a small subset of the FD population in Belgium. ${ }^{12}$

In conclusion, compared with the Rome III approach, the Rome IV classification of FD patients significantly diminishes the overlap group and renders PDS the largest subgroup. Hence, this classification is likely to be more useful in clinical practice for stratifying FD patients, but this needs confirmation in prospective outcome studies.

\section{Uncited Table}

\section{Table 1}

\section{References}

1. Mahadeva S, Goh KL. Epidemiology of functional dyspepsia: a global perspective. World J Gastroenterol 2006;12:2661-2666.

2. Piessevaux H, De Winter B, Louis E, et al. Dyspeptic symptoms in the general population: a factor and cluster analysis of symptom groupings. Neurogastroenterol Motil 2009; 21:378-388.

3. Tack J, Talley NJ, Camilleri M, et al. Functional gastroduodenal disorders. Gastroenterology 2006;130:1466-1479.

4. Tack J, Talley NJ. Functional dyspepsia-symptoms, definitions and validity of the Rome III criteria. Nat Rev Gastroenterol Hepatol 2013;10:134-141.

5. Aro P, Talley NJ, Ronkainen J, et al. Anxiety is associated with uninvestigated and functional dyspepsia (Rome III criteria) in a Swedish population-based study. Gastroenterology 2009; 137:94-100.

6. Zagari RM, Law GR, Fuccio L, et al. Epidemiology of functional dyspepsia and subgroups in the Italian general population: an endoscopic study. Gastroenterology 2010;138:1302-1311.

7. Choung RS, Locke GR, Schleck CD, et al. Do distinct dyspepsia subgroups exist in the community? A population-based study. Am J Gastroenterol 2007;102:1983-1989.

8. van Kerkhoven LA, Laheij RJ, Meineche-Schmidt V, et al. Functional dyspepsia: not all roads seem to lead to Rome. J Clin Gastroenterol 2009;43:118-122.

9. Wang AJ, Liao XH, Xiong LS, et al. The clinical overlap between functional dyspepsia and irritable bowel syndrome based on Rome III criteria. BMC Gastroenterol 2008;8:43.
10. Arts J, Bourgeois S, Claessens C, et al. Discriminant value of dyspepsia subgroups according to the Rome III consensus in dyspeptic patients referred for upper gastrointestinal endoscopy (abstr). Gastroenterology 2008;134(Suppl 1):A-627.

11. Bisschops R, Karamanolis G, Arts J, et al. Relationship between symptoms and ingestion of a meal in functional dyspepsia. Gut 2008;57:1495-1503.

12. Vanheel $H$, Vanuytsel $T$, Van Oudenhove L, et al. Postprandial symptoms originating from the stomach in functional dyspepsia. Neurogastroenterol Motil 2013;25:911-e703.

13. Farré $R$, Vanheel $H$, Vanuytsel $T$, et al. In functional dyspepsia, hypersensitivity to postprandial distention correlates with mealrelated symptom severity. Gastroenterology 2013;145:566-573.

14. Carbone F, Holvoet L, Tack J. Rome III functional dyspepsia subdivision in PDS and EPS: recognizing postprandial symptoms reduces overlap. Neurogastroenterol Motil 2015; 27:1069-1074.

15. Stanghellini V, Chan FK, Hasler WL, et al. Gastroduodenal disorders. Gastroenterology 2016;150:1380-1392.

16. Aziz I, Palsson OS, Törnblom H, et al. Epidemiology, clinical characteristics, and associations for symptom-based Rome IV functional dyspepsia in adults in the USA, Canada, and the UK: a cross-sectional population-based study. Lancet Gastroenterol Hepatol 2018;3:252-262.

17. Van den Houte K, Carbone F, Tack J. Postprandial distress syndrome: stratification and management. Expert Rev Gastroenterol Hepatol 2019;3:337-343.

18. Tack J, Van den Houte K, Carbone F. The unfulfilled promise of prokinetics for functional dyspepsia/postprandial distress syndrome. Am J Gastroenterol 2019;114:204-206.

19. Tack J, Van den Houte K, Carbone F. Gastroduodenal motility disorders. Curr Opin Gastroenterol 2018;34:428-435.

20. Camilleri M, Talley NJ. Pathophysiology as a basis for understanding symptom complexes and therapeutic targets. Neurogastroenterol Motil 2004;16:135-142.

21. Tack J, Bisschops R, Sarnelli G. Pathophysiology and treatment of functional dyspepsia. Gastroenterology 2004;127:1239-1255.

22. Drossman DA, Thompson GW, Talley NJ, et al. Identification of subgroups of functional gastrointestinal disorders. Gastroenterol Int 1990;3:159-172.

23. Talley NJ, Stanghellini V, Heading RC, et al. Functional gastroduodenal disorders. Gut 1999;45(Suppl 2):|I37-II42.

24. Talley NJ, Locke GR, Lahr BD, et al. Predictors of the placebo response in functional dyspepsia. Aliment Pharmacol Ther 2006; 23:923-936.

25. Carbone F, Vanuytsel T, Tack J. Analysis of postprandial symptom patterns in subgroups of patients with Rome III or Rome IV functional dyspepsia. Clin Gastroenterol Hepatol 2020; 18:838-846.

26. Russo F, Chimienti G, Clemente C, et al. Gastric activity and gut peptides in patients with functional dyspepsia: postprandial distress syndrome versus epigastric pain syndrome. J Clin Gastroenterol 2017;51:136-144.

27. Di Stefano M, Miceli E, Tana P, et al. Fasting and postprandial gastric sensorimotor activity in functional dyspepsia: postprandial distress vs. epigastric pain syndrome. Am J Gastroenterol 2014;109:1631-1639.

28. Hsu YC, Liou JM, Yang TH, et al. Proton pump inhibitor versus prokinetic therapy in patients with functional dyspepsia: is therapeutic response predicted by Rome III subgroups? J Gastroenterol 2011;46:183-190. 
29. Suzuki $\mathrm{H}$, Kusunoki $\mathrm{H}$, Kamiya $\mathrm{T}$, et al. Effect of lansoprazole on the epigastric symptoms of functional dyspepsia (ELF study): a multicentre, prospective, randomized, double-blind, placebocontrolled clinical trial. United European Gastroenterol J 2013; $1: 445-452$

30. Carbone F, Vandenberghe A, Holvoet L, et al. 383 - The therapeutic outcome of itopride in functional dyspepsia postprandial distress syndrome: a double-blind randomized, multicenter, placebo-controlled study. Gastroenterology 2018;154:S-91.

\section{Reprint requests}

Address requests for reprints to: Jan Tack, MD, Translational Research Center for Gastrointestinal Diseases (TARGID), University of Leuven, University Hospital Gasthuisberg, O\&N I-Bus 701, Herestraat 49, B-3000 Leuven, Belgium. e-mail: jan.tack@kuleuven.be; fax: (32) 16-34-44-19.

\section{Acknowledgments}

CRediT Authorship Contributions: Jan Tack (Conceptualization: Lead; Data curation: Lead; Formal analysis: Lead; Funding acquisition: Lead; Investigation: Lead; Methodology: Lead; Project administration: Lead; Writing original draft: Lead; Writing - review \& editing: Lead); Karen Van den Houte (Data curation: Equal; Formal analysis: Equal; Investigation: Supporting; Writing - original draft: Lead; Writing - review \& editing: Equal); Florencia Carbone (Conceptualization: Lead; Data curation: Equal; Formal analysis: Equal; Investigation: Equal; Writing - original draft: Equal; Writing - review \& editing: Equal); Nick Goelen (Conceptualization: Equal; Data curation: Equal; Formal analysis: Equal; Investigation: Equal; Writing - original draft: Equal;
Writing - review \& editing: Equal); Jolien Schol (Writing - review \& editing: Equal); Imke Masuy (Writing - review \& editing: Equal); Joris Arts (Data curation: Equal; Investigation: Equal; Writing - review \& editing: Equal); Philip Caenepeel (Data curation: Equal; Investigation: Equal; Writing - review \& editing: Equal); Dirk Staessen (Data curation: Equal; Investigation: Equal; Writing - review \& editing: Equal); Philippe Vergauwe (Data curation: Equal; Investigation: Equal; Writing - review \& editing: Equal); Guy Van Roey (Data curation: Equal; Investigation: Equal; Writing - review \& editing: Equal); Pascale Latour (Data curation: Equal; Investigation: Equal; Writing - review \& editing: Equal); Hubert Piessevaux (Data curation: Equal; Investigation: Equal; Writing - review \& editing: Equal); Philippe Maldague (Data curation: Equal; Investigation: Equal; Writing - review \& editing: Equal); Ariane Gerkens (Data curation: Equal; Investigation: Equal; Writing - review \& editing Equal); Fabien Wuestenberghs (Data curation: Equal; Investigation: Equal; Writing - review \& editing: Equal); Alain Vandenberghe (Conceptualization: Lead; Data curation: Equal; Investigation: Equal; Writing - review \& editing: Equal).

\section{Conflicts of interest}

This author discloses the following: Jan Tack has given scientific advice to AlfaWassermann, Allergan, Christian Hansen, Danone, Grünenthal, Ironwood, Janssen, Kiowa Kirin, Menarini, Mylan, Neutec, Novartis, Noventure, Nutricia, Shionogi, Shire, Takeda, Theravance, Tramedico, Truvion, Tsumura, Zealand, and Zeria Pharmaceuticals, has received research support from Shire, Sofar, and Tsumura, and has served on the speakers bureau for Abbott, Allergan, AstraZeneca, Janssen, Kyowa Kirin, Menarini, Mylan, Novartis, Shire, Takeda, Truvion, and Zeria. The remaining authors disclose no conflicts.

\section{Funding}

This study was supported by a research grant from Zeria Pharmaceuticals. 